\title{
RADIOGRAPHIC CARIES DETECTION BEHAVIOR IN A SAMPLE OF DENTISTS AND DENTAL SPECIALISTS
}

\author{
Emad A. Khan*
}

\begin{abstract}
Radiographic caries detection depends not only on correctly acquired radiographs but also on the way they are displayed and interpreted.

Objective: This research aims to measure behavioral factors important for caries detection among dentists.

Materials \& Methods: A sample of 206 dentists was surveyed using a self-reported questionnaire after a focus group and a pilot study. Questions asked were related to the type of image used, the way the image is displayed, the use of magnification, and the use of vision correction aids if needed. Responses were analyzed using descriptive statistics.
\end{abstract}

Results: The use of the following was BW (>95\%), viewing box $(68 \%)$, masking extra-light $(<15 \%)$, dimming room's light $(<15 \%)$, magnification $(<13 \%)$, and vision correction aid $(85 \%$ from those who need it). The most frequently reported reasons were "availability" and "lack of knowledge".

Conclusions: There is a significant undesirable behavioral practice during radiographic caries detection among the studied sample. The main reasons seem to be "convenience" and lack of knowledge.

\section{INTRODUCTION}

Caries diagnosis is a very complex task. Radiographic detection is one of the important diagnostic tools for caries diagnosis. It's by itself another complex and multifactorial task. For accurate diagnosis, radiographs should be acquired correctly. However, this is not enough. These good radiographs could lead to misdiagnosis if not viewed and interpreted correctly. ${ }^{1}$ Optimal viewing conditions are emphasized in oral radiology textbooks following the ADA recommendations. ${ }^{2}$ Some literature have for a long time focused on studying those viewing conditions to identify important ones and how the observers approach these factors. ${ }^{3-5}$

* King Abdulaziz University - Faculty of Dentistry (KAUFD) 
In our population of dentists, radiographic caries detection behavior has not been studied. Our aim is to identify potential behavioral practice that could lead to less than optimal caries diagnosis, which is a national priority.

\section{MATERIAL AND METHOD}

A self-reported questionnaire composed of 9 questions in addition to demographic data, has been distributed to 206 mainly general and restorative dentists but also other dental specialists, randomly selected from 3 different major hospitals in the city. Each completed questionnaire has been collected at the same day it was handed to the dentist. The time needed to complete the questionnaire was approximately 5 minutes.

The questions focused on the behavior and practice of dentists during radiographic caries detection including the type of image used (bitewing (BW), periapical (pa) or panoramic radiographs), the way of displaying the image and some details about it, the use of magnification, and the use of vision correction aids if needed. These questions were formulated after a focus group of a radiologist, a restorative dentist and 2 general dentists discussed important modifiable behavioral factors post-image acquisition. A pilot questionnaire was distributed to 5 dentists and the feedback was used to finalize the study questionnaire. Responses were analyzed and compared using descriptive statistics.

\section{RESULTS}

Our sample consisted of 92 male and 114 female dentists, mostly (90\%) general dentists, of which 161 were new graduates (interns). The age range was 22-46 years.

When asked if they use BW for radiographic caries detection, the majority agreed while only 10 said "not always" and the main reason was "time restriction". One dentist had a concept of "Panoramic radiograph is sufficient".
Regarding the use of a viewing box, 66 (32\%) don't always use it because of mainly "availability". One dentist expressed that "the ceiling light is sufficient". When asked if they use a "clean viewing box", 76 (37\%) responded negatively and the main reason was also "availability". We asked them if they mask the extra-light and the majority $(74.8 \%)$ said "No" in addition to $24(11.65 \%)$ who responded: "Not always". The main reason mentioned was "I didn't know I should". More than half of the dentists (52.9\%) don't dim the light while detecting caries and $33.5 \%$ not always do. The reason mentioned was "feasibility".

Regarding magnification, also more than half of the sample (55.3\%) don't use magnification and $32 \%$ not always do, with the "availability" as the main reason. It was found that 92 dentists $(44.7 \%)$ reported a need for vision correction aid (eyeglasses or contact lenses), from those, 11 don't use them and 4 "not always" do during caries detection.

\section{DISCUSSION}

BW is still the standard of care for radiographic caries detection. It has been shown that it is superior to panoramic radiography although some special acquisition mode and digital enhancement might improve the performance of it. ${ }^{6} \mathrm{BW}$ is also superior to pa in caries detection. ${ }^{7}$ Results from this study show that most dentists know and practice caries detection using BW. A minority might make a mistake of relying on less than optimum diagnostic tools and the reason for this is either "time" or lack of knowledge, neither of which is acceptable if a diagnostic error is made.

A properly exposed and processed film tends to be classified as "dark". Studies have shown that radiographs give better diagnostic information when viewed on a viewing box with masking the extra-light. Viewing box and masking of extralight have been recommended and tested for better diagnostic performance when interpreting intra- 
oral radiographs, especially for difficult to detect diseases like periapical inflammatory pathosis. ${ }^{8}$ It is especially important to mask extra-light from the viewing box more than using a viewing box. Viewing box without masking could be comparable to ceiling light and that gives reasonable diagnostic information if the film is light. ${ }^{3}$ Masking could be done using commercially available tools with or without a magnifying lens. The majority of our sample did not give enough importance to having a viewing box, clean ones, or masking extralight, which indicates a potential for inaccurate or incomplete caries diagnosis.

Bright rooms can reduce the image contrast and theoretically may affect caries detection. However, background lighting has been shown to be of insignificance regarding its effect on the ability to detect caries..$^{9,10}$ A minority of our sample reported that they do dim the light during radiographic caries detection.

Magnification has been reported to increase caries detection accuracy and improve diagnostic performance of small lesions diagnosis. ${ }^{8,11}$ A large majority (more than $85 \%$ ) of surveyed dentists were not very careful to use magnification for radiographic caries detection. This indicates another area of potential diagnostic deficiency. The good news is that the main reason was "availability" of magnifying lens. It could be assumed that this will improve with the introduction of digital imaging since magnification is readily available.

One of the main factors in the accuracy of radiographic caries detection is the observer. Observer's visual acuity is of primary importance. Not using vision correction aids when needed has a great potential for less diagnostic performance. Results show that a large number of surveyed dentists need vision correction aids and fortunately, only a small number don't always use them during caries detection.
Analyzing the different reported reasons for undesirable behavior reveals that "convenience" and lack of knowledge represent the main problem.

Based on these findings we recommend a more emphasis on the basic concepts of caries detection that are deficient in this sample - during educational process, and a robust quality control and clinical standard of care during clinical practice of caries management, to improve diagnosis and prevent potential risks during caries management process.

\section{CONCLUSION}

A number of important behavioral factors for radiographic caries detection have been studied. There is a need for more education and practice monitoring to improve diagnostic performance and prevent potential risks of diagnostic deficiencies.

\section{ACKNOWLEDGEMENT}

Dr. Lujain Homeida, Umm Al-Qura University, and Dr. Alaa Nadhreen, King Abdulaziz University, for their help collecting the data.

\section{REFERENCES}

1. Wuehrmann AH. Radiation hygiene and its practice in dentistry as related to film viewing procedures and radiographic interpretation. Council on Dental Materials and Devices. J Am Dent Assoc 1970;80(2):346-56.

2. White SC, Pharoah MJ. White and Pharoah's Oral Radiology E-Book: Principles and Interpretation: Elsevier Health Sciences; 2018.

3. Welander U, McDavid WD, Higgins NM, Morris CR. The effect of viewing conditions on the perceptibility of radiographic details. Oral Surg Oral Med Oral Pathol 1983;56(6):651-4.

4. Mattsson O. Aspects of the interpretation of contrast and detail in radiographs. Acta radiol 1952;38(6):477-88.

5. Espelid I. The influence of viewing conditions on observer performance in dental radiology. Acta Odontol Scand 1987;45(3):153-61. 
6. Khan EA, Tyndall DA, Caplan D. Extraoral imaging for proximal caries detection: Bitewings vs scanogram. Oral Surg Oral Med Oral Pathol Oral Radiol Endod 2004;98(6):730-7.

7. Takahashi N, Lee C, Da Silva JD, et al. A comparison of diagnosis of early stage interproximal caries with bitewing radiographs and periapical images using consensus reference. Dentomaxillofac Radiol 2019;48(2):20170450.

8. Patel N, Rushton VE, Macfarlane TV, Horner K. The influence of viewing conditions on radiological diagnosis of periapical inflammation. Br Dent J 2000;189(1):40-2.
9. Cederberg RA, Frederiksen NL, Benson BW, Shulman JD. Effect of different background lighting conditions on diagnostic performance of digital and film images. Dentomaxillofac Radiol 1998;27(5):293-7.

10. Deep P, Petropoulos D. Effect of illumination on the accuracy of identifying interproximal carious lesions on bitewing radiographs. J Can Dent Assoc 2003;69(7):444-6.

11. Arnold LV. The radiographic detection of initial carious lesions on the proximal surfaces of teeth. Part II. The influence of viewing conditions. Oral Surg Oral Med Oral Pathol 1987;64(2):232-40. 